EDITORIAL

\title{
Regional variation in ICD implantation rates: the shocking truth?
}

C C Lang

Heart 2005;91:1251-1253. doi: 10.1136/hrt.2004.056754

There is considerable disparity in the implantation of ICDs across the UK, with a fourfold difference between regions with the highest and lowest implantation rates

I

$\mathrm{n}$ this issue of Heart, Cunningham et al present audit data from the National Pacemaker Database for the period 1998-2002 detailing the implantation rates of implantable cardioverter-defibrillators (ICDs) and show that the UK as a whole is implanting fewer ICDs than many western European countries. This article represents an update on previous reports with similar findings. ${ }^{2}{ }^{3}$ In addition to these national comparisons, the authors break down implantation rates by region and demonstrate considerable disparity, with a fourfold difference between regions with the highest and lowest rate of ICD implantation.

Since the introduction of ICDs into clinical practice, considerable evidence from controlled randomised trials has been produced supporting their use in both secondary and primary prevention settings. In particular, the utility of ICDs has been shown in patients with ischaemic heart disease and left ventricular impairment, who represent the majority of patients suffering sudden cardiac death. Guidelines for England and Wales were produced in 2000 by the National Institute for Clinical Excellence (NICE) based on the trial data available at that time, stipulating criteria that should be fulfilled for a patient to receive a device. ${ }^{4}$ This document suggested that an implantation rate of around 50 per million population per year could be expected.

The data presented by Cunningham spans a transitional period in terms of clinical practice, including the publication of the NICE guidelines. As expected, over the study period there was a rise in implantation rates in all four countries of the UK. Northern Ireland was the only country to achieve the implant rate predicted by NICE of 50 per million per year. The range of implantation rates seen within the regional "league table" in Cunningham's article requires further investigation and scrutiny. The observed differences are unlikely to be explained by physician's attitudes

Correspondence to: Dr Christopher C Lang, Department of Cardiology, Royal Infirmary of Edinburgh, Little France Crescent, Edinburgh EH16 4SA, UK; chrislang@ katamail.com to, or execution of, the NICE guidelines.

\section{PRIMARY VERSUS SECONDARY PREVENTION?}

Until relatively recently, ICDs were largely reserved for patients surviving an out of hospital threatening arrhythmias while on amiodarone treatment. Subsequently, study data demonstrated the superiority of ICDs compared to antiarrhythmic treatment, particularly when left ventricular function is impaired (the AVID ${ }^{5}$ and CIDS $^{6}$ trials), encouraging us to consider device therapy as the first choice in these patients. The MADIT $^{7}$ and MUSTT $^{8}$ trials provided support for the use of ICDs for the primary prevention of sudden death in patients with ischaemic heart disease and impaired left ventricular function found to have inducible ventricular arrhythmias at electrophysiological (EP) testing. Based on MADIT data, the NICE guidelines stipulated that patients with previous myocardial infarction, a non-sustained ventricular tachycardia on Holter, and an ejection fraction of less than 35\% should be considered for an ICD if sustained ventricular tachycardia can be induced during EP testing. These criteria defined the national therapeutic indications for ICD, and a rise in implantation rates was expected. The observed increase in implantation rates has been slow, with considerable variation across the UK. Several factors need to be considered in order to understand the observed variation.

\section{SECONDARY PREVENTION}

Patients with life threatening arrhythmias and ischaemic heart disease account for around 75\% of all individuals with a secondary prevention indication for ICD therapy. Cunningham et $a l^{1}$ state that the regional variation in implantation rate cannot be explained by differences in survival from cardiac arrest. However, there is evidence that the likelihood of surviving OOHCA does indeed vary widely, and is dependent on local factors such as the distance from the arrest location to the nearest ambulance station. For example, the probability of survival to hospital admission varies more than fourfold between the hinterland of Edinburgh and the city centre," with the distance between arrest location and ambulance station being a key factor; this suggests that less densely populated regions would have a significantly lower OOHCA survival rate than regions where the population is predominantly city based. In addition, comparisons between data from North Glasgow and cardiac arrest (OOHCA), or having had life

Abbreviations: $A V I D$, antiarrhythmics versus implantable defibrillators; CIDS, Canadian implantable defibrillator study; EP, electrophysiology; ICD, implantable cardioverter-defibrillator; MADIT, multicentre automatic defibrillator implantation trial; MUST, multicenter unsustained tachycardia trial; NICE, National Institute for Clinical Excellence; OOHCA, out of hospital cardiac arrest 
Edinburgh from a similar period in time show at least a fourfold variation in survival to discharge rates. ${ }^{9}{ }^{10}$

\section{PRIMARY PREVENTION}

By and large, patients with a secondary prevention indication are readily identifiable. However, patients with primary prevention indications (as defined by NICE) are harder to recognise. To identify "at risk" patients there must be a comprehensive screening programme of patients with previous myocardial infarction which includes not only the assessment of left ventricular function (which should ideally be done more than one month from the time of the infarct), but also the presence of non-sustained ventricular tachycardia on Holter monitoring. Patients fulfilling these criteria then have to be referred for invasive EP testing. The identification of these patients requires a change in practice for the follow up of patients, and EP testing will in most cases require referral or transfer to a tertiary centre. The selection criteria therefore decrease the probability of identification through their complexity and the resource implications.

Patients identified and referred for ICD implantation for primary prevention represent the tip of the iceberg in terms of the number that would actually benefit from such therapy, and an implantation rate of 50 per million is a conservative estimate. ${ }^{11}$ That some regions are only reaching a fifth of this rate suggests problems identifying these patents. Cardiology departments need to consider integrating screening for arrhythmic risk into post-myocardial infarction follow up and heart failure clinics in order to increase detection rates.

The trends in the relative proportion of primary and secondary prevention ICD recipients would be helpful to understand whether the NICE guidelines were being embraced and successfully implemented, both nationally and regionally. One might expect that the increase in implantation rate would be largely accounted for by an increase in primary prevention implants, as a dramatic increase in survival following OOHCA is not likely to occur without major changes in the delivery of out of hospital resuscitation care.

\section{RESOURCE IMPLICATIONS: MORE CENTRES OR BIGGER CENTRES?}

There has been a dramatic increase in the number of consultant cardiologists in England due to additional funding for the management of cardiac disease through the National Service Framework. This will have had a positive impact on the management of patients with ischaemic heart disease and possibly in the identification and management of patients at risk of arrhythmias. The variations in national and regional implantation data may reflect the distribution of cardiologists. It would be useful to correlate the implantation rates with the distribution of electrophysiologists or device specialists. Running a comprehensive ICD service is costly and staff intensive, and an increase in implantation rates can only be achieved through the provision of adequate resources in terms of screening patients, device costs, and technical support for follow up.

There is no practical reason why in principle, as with bradycardia pacing, ICD implantation should be confined to tertiary centres. Technically, implanting a modern defibrillator requires little additional experience to that already possessed by a cardiologist who performs pacing. Devices are now small enough that the implantation technique is virtually identical to that of a bradycardia pacemaker. VF induction and defibrillation is performed under conscious sedation rather than general anaesthesia, and the precautions necessary to perform this safely are the same as when sedation is employed for transoesophageal echocardiography. Although initial programming and follow up of these devices requires training and careful thought, device companies run frequent excellent courses to support physicians and technicians in this area.

\section{DATA COLLECTION AND MONITORING}

For many years, we have been collecting data on coronary angiography and intervention using the Minerva database. This database provides data on patient demographics, risk factors, indications, complications, and outcomes. At present, there is no such system in place for pacemakers and defibrillators. The data collected by the National Pacemaker Database is invaluable, as the article by Cunningham et al ${ }^{1}$ demonstrates. It would be extremely useful to extend the scope of data collection for ICDs to include primary and secondary prevention indications, and more detail on patient characteristics, as is the case with coronary intervention. In addition, as recommended by NICE, all cardiology units must audit their practice in order to estimate demand and take steps to secure resources. It will also be important to collect data on the incidence of patients fulfilling the criteria used in the later primary prevention trials such as MADIT-II ${ }^{12}$ and SCD-HeFT, ${ }^{13}$ as the outcomes of these studies will influence future guidelines and it will be advantageous to appreciate in advance the implications for service provision.

\section{THE FUTURE?}

NICE is due to issue new guidance on ICD therapy in coming months. It is likely that the results of the more recent secondary prevention study MADIT-II will influence selection criteria, which would simplify the identification of patients by removing the need for EP testing, but at the same time increasing the number of eligible patients.

It is essential that we acknowledge regional trends in ICD implantation and seek explanations for the observations. The data presented by Cunningham et $a l^{1}$ is now more than two years out of date, and more frequent data collection and reporting is necessary to keep abreast of the situation. Managed clinical networks will facilitate regional service provision, helping to resolve issues and assist in service expansion. As with interventional cardiology, an expansion in the number of "device specialists" will be necessary, and not only in major teaching hospitals. Hopefully, the article by Cunningham et al will provide the stimulus for change in this important area.

\section{REFERENCES}

1 Cunningham AD, Plummer CJ, McComb JM, et al. The implantable cardioverter-defibrillator: postcode prescribing in the UK 1998-2002. Heart 2005:91:1280-3.

2 Camm AJ, Nisam S. The utilization of the implantable defibrillator - a European enigma. Eur Heart J 2000;21:1998-2004.

3 Seidl K, Senges J. Geographic differences in implantable cardioverter defibrillator usage. J Cardiovasc Electrophysiol 2002;13(suppl 1):S100-5.

4 National Institute for Clinical Excellence. Technology Appraisal guidance No 11. Guidance on the use of implantable cardioverter defibrillators for arrhythmias. London: NICE, September, 2000.

5 The AVID Investigators. A comparison of anti-arrhythmic drug therapy with implantable defibrillators in patients resuscitated from near fatal ventricular arrhythmias. N Engl J Med 1997;337:1576-83.

6 Connolly SJ, Gent M, Roberts RS, et al. Canadian implantable defibrillator study (CIDS): a randomized trial of the implantable cardioverter defibrillato against amiodarone. Circulation 2000;101:1297-302.

7 Moss AJ, Hall J, Cannom DS, et al for the Multicentre Automatic Defibrillator Implantation Trial (MADIT) Investigators. N Engl J Med 1996;335:1933-40.

8 Buxton AE, Lee KL, Fisher JD, et al for the Multicenter Unsustained Tachycardia Trial (MUSTT) Investigators. A randomized study of sudden death in patients with coronary artery disease. N Engl J Med 1997:341:1882-90.

9 Lyon RM, Cobbe SM, Bradley JM, et al. Surviving out of hospital cardiac arrest at home: a postcode lottery? Emerg Med 2004;21:619-24.

10 Leslie WS, Fitzpatrick B, Morrison CE, et al. Out-of-hospital cardiac arrest due to coronary heart disease: a comparison of survival before and after the introduction of defibrillators in ambulances. Heart 1996:75:195-9.

11 Plummer CJ, Irving RJ, McComb JM. Implications of national guidance for implantable cardioverter defibrillation implantation in the United Kingdom. Pacing Clin Electrophysiol 2003;26(pt II):479-82. 
12 Moss AJ, Zareba W, Hall WJ, et al for the MADIT II Investigators. Prophylactic Implantation of a defibrillator in patients with myocardial infarction and reduced ejection fraction. N Engl J Med 2002;346:877-83.
13 Brady GH, Lee KL, Mark DB et al for the SCD-HeFT Investigators Amiodarone or an implantable cardioverter defibrillator for congestive heart failure. N Engl J Med 2005;352:225-37.

\section{IMAGES IN CARDIOLOGY}

\section{Intramyocardial septal branches of a "dual LAD" selectively visualised within a no reflow area}

A

75 year old man was admitted to our coronary care unit with anterolateral ST elevation myocardial infarction (STEMI). After intravenous treatment with aspirin and nitrates, the patient underwent urgent coronary angiography that documented occlusion of the proximal left anterior descending coronary artery (LAD), with TIMI 0 flow (panel A). After crossing the lesion with the guidewires, a large thrombus became evident (panel B). During the procedure "dual LAD" anatomy was documented with a bifurcation of the LAD into a large septal branch (with intramyocardial course) and a large diagonal branch (panel C). A two dimensional echocardiogram documented a slightly hypertrophied and dilated left ventricle, with akinesia of the apex, mid anterior wall, lateral wall, and interventricular septum, and hypokinesia of relative basal segments with an ejection fraction of $35 \%$. Twelve hours after percutaneous coronary intervention, a myocardial contrast echo study was performed using a Sequoia ultrasound machine (Siemens) and intravenous SonoVue (Bracco) $5 \mathrm{ml}$ at $2 \mathrm{ml} / \mathrm{min}$. A novel contrast detection method, called contrast pulse sequencing (CPS), was applied to visualise the coronary microcirculation. This method is able to selectively detect in real time the nonlinear, fundamental ultrasound frequency, that, being particularly strong and peculiar to microbubbles, gives a high intensity signal with excellent signal-to-noise ratio. The patient had a large area of microvascular obstruction (no reflow) at the level of the apex, septum, and posterolateral wall (panels D and E). Within this no reflow area, septal and apical branches likely originated from the intramural septal branch of the dual LAD and were visible as yellow lines crossing the wall from the epicardium to the endocardium (panels D and E).
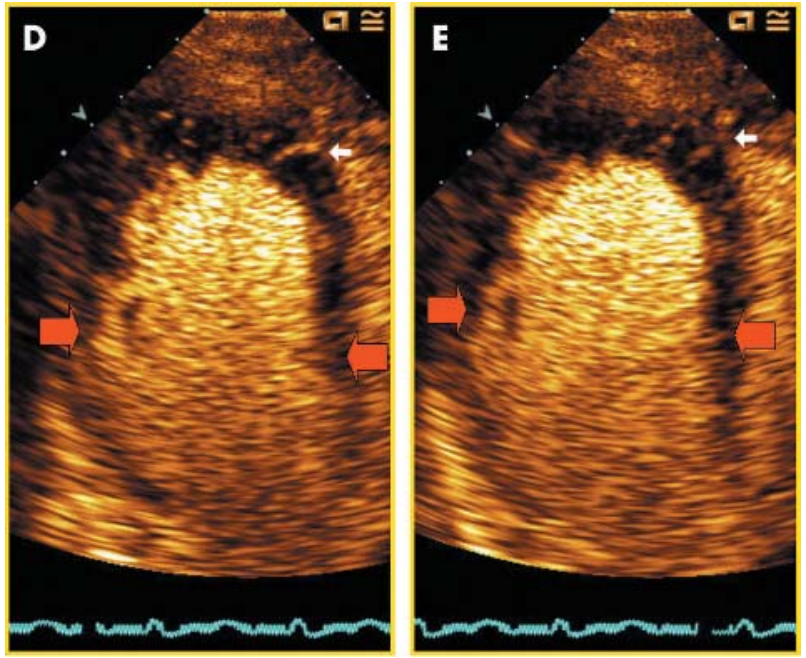

Myocardial contrast echo consecutive frames (panel D and E) in apical long axis view showing a large no reflow area at the level of the interventricular septum, apex, and posterolateral wall (within red arrows). Intramyocardial perforator branches are visible as yellow lines crossing the wall from the epicardium to the endocardium at the level of the apical septum (white arrow).
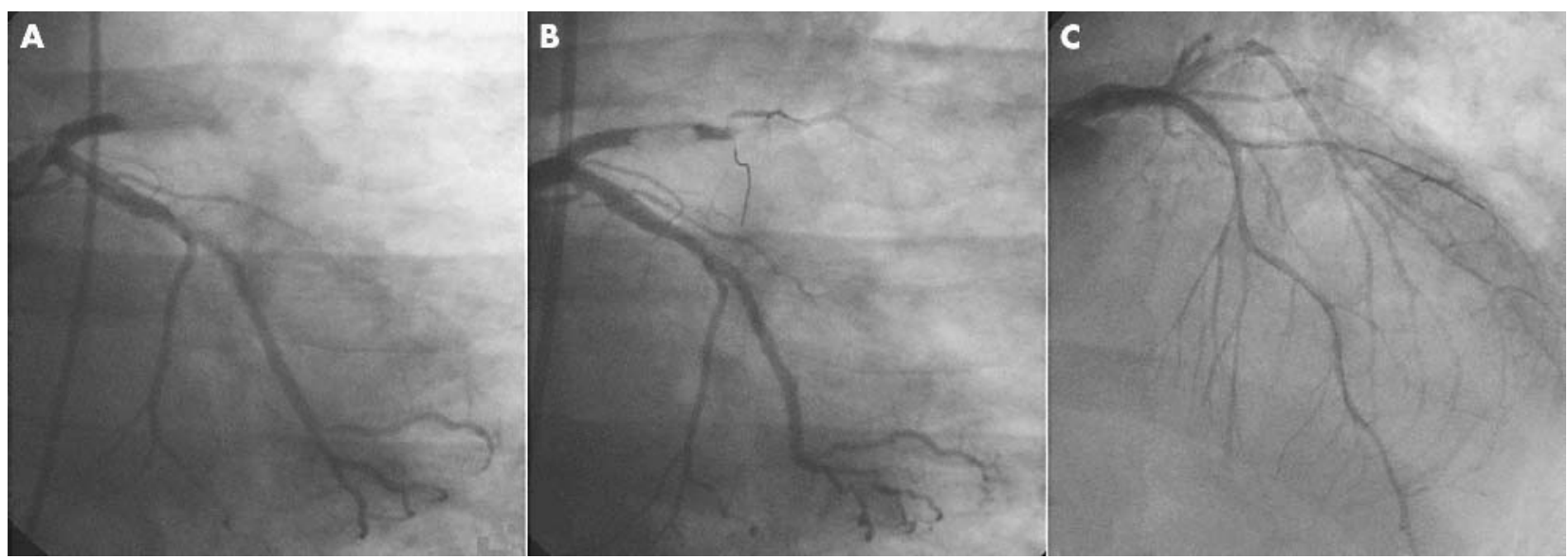

Coronary angiogram showing total occlusion of proximal left anterior descending (LAD) coronary artery in caudal right anterior oblique (RAO) view (panel A). In the same view, after crossing the LAD lesion with the guidewires, a large thrombus was evident (panel B). Post-stenting, a coronary angiogram in cranial RAO view showed a "dual LAD" anatomy (two guidewires inside the septal and the diagonal branches) (panel C). 